\title{
Promise of periodontal ligament stem cells in regeneration of periodontium
}

\author{
Hidefumi Maeda*, Atsushi Tomokiyo, Shinsuke Fujii, Naohisa Wada and Akifumi Akamine
}

\begin{abstract}
A great number of patients around the world experience tooth loss that is attributed to irretrievable damage of the periodontium caused by deep caries, severe periodontal diseases or irreversible trauma. The periodontium is a complex tissue composed mainly of two soft tissues and two hard tissues; the former includes the periodontal ligament (PDL) tissue and gingival tissue, and the latter includes alveolar bone and cementum covering the tooth root. Tissue engineering techniques are therefore required for regeneration of these tissues. In particular, PDL is a dynamic connective tissue that is subjected to continual adaptation to maintain tissue size and width, as well as structural integrity, including ligament fibers and bone modeling. PDL tissue is central in the periodontium to retain the tooth in the bone socket, and is currently recognized to include somatic mesenchymal stem cells that could reconstruct the periodontium. However, successful treatment using these stem cells to regenerate the periodontium efficiently has not yet been developed. In the present article, we discuss the contemporary standpoints and approaches for these stem cells in the field of regenerative medicine in dentistry.
\end{abstract}

Periodontal ligament stem cells (PDLSCs) represent a promising cell-based therapy in reconstructive dentistry for the treatment of damaged periodontium. Researchers have therefore attempted to identify PDLSCs and disclose their characteristics. In the 1980s, the cells that exhibited small size, a high nuclear/cytoplasmic ratio and slow cell division were reported to be localized in regions adjacent to blood vessels, and these cells were suggested as PDLSCs [1,2]. In 2004, human PDLSCs exhibiting

*Correspondence: hide@dent.kyushu-u.ac.jp

Department of Endodontology \& Operative Dentistry, Kyushu University, 3-1-1 Maidashi, Fukuoka 812-8582, Japan self-renewal and multipotent capacities were first isolated from human periodontal ligament (PDL) tissue [3].

PDLSCs exhibit unique properties. This group of authors and others have demonstrated the plasticity of PDLSCs to differentiate into osteoblastic and adipocytic cells $[4,5]$. Furthermore, Seo and colleagues identified STRO-1 and CD146 as potent surface markers of PDLSCs [3]. The percentage of cells resident in PDL tissue positive for these markers is very low, however, indicative of the difficulty in acquiring a sufficient number of these cells from a patient for clinical use. Yet recently a clinical trial using proliferative human PDL cells including PDLSCs was conducted on patients with infrabony defects, revealing a significant improvement of periodontal diseases, and suggesting that cell transplantation could be a safe and promising treatment [6]. In addition, an in vitro study revealed that collagen and a synthetic polymer are useful scaffolds for PDLSC survival [7], also suggesting the feasibility of long-term analysis. However, the issue related to number of PDL cells available for regenerative treatment in clinical practice remains unresolved.

As alternative stem cells to PDLSCs, bone marrow mesenchymal stem cells (BMMSCs) have been a focus of attention to resolve the above issue because PDLSCs also express other surface markers similar to BMMSCs, such as CD9, CD10, CD13, CD29, CD44, CD49d, CD90, CD105, CD146, and CD166. Zhou and colleagues revealed the possible participation in periodontal healing of allogenic BMMSCs transplanted into irradiated mice [8]. Yang and colleagues indicated that BMMSCs were beneficial source cells, revealing the improvement of periodontal defects experimentally created in rats when transplanted directly with microcarrier gelatin beads [9].

Adipose-derived stem cells are also indicated to have probable advantages for tissue engineering applications because of their multipotency and convenient isolation in large amounts without pain for donors. The application of adipose-derived stem cells into periodontal defects in rats suggested a potential contribution to tissue healing [10]. Murine induced pluripotent stem cells that were applied with enamel matrix derivative to periodontal defects in mice implied a capability to promote periodontal regeneration [11]. These stem cells may thus have 
the feasibility to reconstruct the damaged periodontium, probably by communicating with surrounding dental tissue. However, the mechanism to direct these stem cells towards periodontal cells remains unclear.

In this context, researchers have focused attention on dental follicle cells (DFCs) in an effort to address this condition because DFCs are believed to be parent cells that differentiate into PDL fibroblasts as well as osteoblasts and cementoblasts fabricating cementum during development of the periodontium [12]. In the past few years, there has been energetic progress in characterizing DFCs to clarify how they were controlled during differentiation into these cells. GoPro49, a novel Golgi protein, was identified as a specific marker for DFCs [13]. Morsczeck and colleagues reported the transcriptomes and proteomes of human DFCs in relationship with osteoblastic/cementoblastic or fibroblastic differentiation [14]. Dangaria and colleagues suggested the contribution of signature gene expression, unique shifts in gene cohort expression levels, epigenetic modifications, and changes in cell morphology to the differentiation of DFCs [15]. Most recent reports have suggested the involvement of Hertwig's epithelial root sheath cells (HERSCs) in the differentiation of DFCs $[16,17]$. Since HERSCs are epithelial cells that play an important role in development of tooth root, these HERSCs may also contribute to fabrication of the periodontium. Although what is central to regulating the optimum differentiation of DFCs remains uncertain, as growth of dental tissues is attributable to epithelial-mesenchymal interaction comprehensively, hereafter it may become a critical issue to elucidate participation of such epithelial cells in periodontal regeneration as well as periodontal development.

There are other trends focusing on stem cells resident in PDL tissue (that is, PDLSCs themselves) to elucidate their involvement in the regenerative process, and to develop attractive and novel regenerative treatment techniques. For these purposes several groups have attempted to establish immortalized clonal PDLSC lines for convenient and routine analyses. Our group for the first time succeeded in establishing two human clonal PDLSC lines with multipotency by transducing primary PDL cells with both SV40 large T-antigen and human telomerase reverse transcriptase genes [18-20], whereas another group presented immortalized PDLSCs by transducing bone morphogenetic protein 4 and human telomerase reverse transcriptase genes [21]. Our developed clones exhibit different characteristics from each other in multipotency, expression of stem cell makers, responsiveness to growth factors such as basic fibroblast growth factor and enamel matrix derivative, and the expression of bone morphogenetic protein 4 and fibroblast growth factor receptor 1 - suggesting that PDLSCs at diverse differentiation stages are localized in PDL tissue and that these clones were definitely derived from such PDLSCs.

We therefore believe that differential analyses between these two clones will allow us to further clarify the mechanism of PDLSC and even DFC differentiation. These results will enable elucidation of signals that direct stem cells including BMMSCs, induced pluripotent stem cells or embryonic stem cells toward PDL-lineage cells, and furthermore to identify the optimum signaling molecules and scaffolds for the periodontium regeneration, including potential signals from HERSCs. By integrating these constituents, we will be able to develop a novel regenerative medicine of the periodontium. Since complex tissue regeneration including two hard tissues and two soft tissues is required for reconstruction of the periodontium, these novel tissue engineering techniques will make it possible to develop innovative regenerative medicines with a wide field of systemic application.

\section{Abbreviations \\ BMMSC, bone marrow mesenchymal stem cell; DFC, dental follicle cell; HERSC, Hertwig's epithelial root sheath cell; PDL, periodontal ligament; PDLSC, periodontal ligament stem cell.}

Competing interests

The authors declare that they have no competing interests.

\section{Acknowledgements}

The authors thank Dr Monnouchi, Dr Kono, Dr Koori, Dr Yamamoto and Dr Teramatsu for their great support in preparation of this article. The present work was financially supported by grants-in-aid (Project Numbers 21390510 , 22390359, 22592123, and 23659890) for scientific research from the Ministry of Education, Culture, Sports, Science and Technology (Japan).

Published: 28 July 2011

\section{References}

1. Gould TR: Ultrastructural characteristics of progenitor cell populations in the periodontal ligament. J Dent Res 1983, 62:873-876.

2. McCulloch CA: Progenitor cell populations in the periodontal ligament of mice. Anat Rec 1985, 211:258-262.

3. Seo BM, Miura M, Gronthos S, Bartold PM, Batouli S, Brahim J, Young M, Robey $P G$, Wang CY, Shi S: Investigation of multipotent postnatal stem cells from human periodontal ligament. Lancet 2004, 364:149-155.

4. Huang CY, Pelaez D, Dominguez-Bendala J, Garcia-Godoy F, Cheung HS: Plasticity of stem cells derived from adult periodontal ligament. Regen Med 2009, 4:809-821.

5. Chadipiralla K, Yochim JM, Bahuleyan B, Huang CY, Garcia-Godoy F, Murray PE, Stelnicki EJ: Osteogenic differentiation of stem cells derived from human periodontal ligaments and pulp of human exfoliated deciduous teeth. Cell Tissue Res 2010, 340:323-333.

6. Feng F, Akiyama K, Liu Y, Yamaza T, Wang TM, Chen JH, Wang BB, Huang GT, Wang S, Shi S: Utility of PDL progenitors for in vivo tissue regeneration: a report of 3 cases. Oral Dis 2010, 16:20-28.

7. Gebhardt M, Murray PE, Namerow KN, Kuttler S, Garcia-Godoy F: Cell survival within pulp and periodontal constructs. J Endod 2009, 35:63-66.

8. Zhou J, Shi S, Shi Y, Xie H, Chen L, He Y, Guo W, Wen L, Jin Y: Role of bone marrow-derived progenitor cells in the maintenance and regeneration of dental mesenchymal tissues. J Cell Physio/ 2011, 226:2081-2090.

9. Yang Y, Rossi FM, Putnins EE: Periodontal regeneration using engineered bone marrow mesenchymal stromal cells. Biomaterials 2010, 31:8574-8582.

10. Tobita M, Uysal AC, Ogawa R, Hyakusoku H, Mizuno H: Periodontal tissue regeneration with adipose-derived stem cells. Tissue Eng Part A 2008, 14:945-953.

11. Duan X, Tu Q, Zhang J, Ye J, Sommer C, Mostoslavsky G, Kaplan D, Yang P, Chen J: Application of induced pluripotent stem (iPS) cells in periodontal 
tissue regeneration. J Cell Physio/ 2011, 26:150-157.

12. Luan X, Dangaria S, Ito Y, Walker CG, Jin T, Schmidt MK, Galang MT, Druzinsky $R$ : Neural crest lineage segregation: a blueprint for periodontal regeneration. J Dent Res 2009, 88:781-791

13. Takatalo MS, Tummers M, Thesleff I, Rönnholm R: Novel Golgi protein, GoPro49, is a specific dental follicle marker. J Dent Res 2009, 88:534-538.

14. Morsczeck C, Schmalz G, Reichert TE, Völlner F, Saugspier M, Viale-Bouroncle S, Driemel O: Gene expression profiles of dental follicle cells before and after osteogenic differentiation in vitro. Clin Oral Investig 2009, 13:383-391.

15. Dangaria SJ, Ito Y, Luan X, Diekwisch TG: Differentiation of neural-crestderived intermediate pluripotent progenitors into committed periodontal populations involves unique molecular signature changes, cohort shifts, and epigenetic modifications. Stem Cells Dev 2011, 20:39-52.

16. Jung HS, Lee DS, Lee JH, Park SJ, Lee G, Seo BM, Ko JS, Park JC: Directing the differentiation of human dental follicle cells into cementoblasts and/or osteoblasts by a combination of HERS and pulp cells. J Mol Histol 2011, 42:227-235.

17. Bai Y, Bai Y, Matsuzaka K, Hashimoto S, Fukuyama T, Wu L, Miwa T, Liu X, Wang $X$, Inoue $T$ : Cementum- and periodontal ligament-like tissue formation by dental follicle cell sheets co-cultured with Hertwig's epithelial root sheath cells. Bone 2011, 48:1417-1426
18. Fujii S, Maeda H, Wada N, Kano Y, Akamine A: Establishing and characterizing human periodontal ligament fibroblasts immortalized by SV40T-antigen and hTERT gene transfer. Cell Tissue Res 2006, 324:117-125.

19. Tomokiyo A, Maeda H, Fujii S, Wada N, Shima K, Akamine A: Development of a multipotent clonal human periodontal ligament cell line. Differentiation 2008, 76:337-347.

20. Fujii S, Maeda H, Wada N, Tomokiyo A, Saito M, Akamine A: Investigating a clonal human periodontal ligament progenitor/stem cell line in vitro and in vivo. J Cell Physio/ 2008, 215:743-749.

21. Mi HW, Lee MC, Fu E, Chow LP, Lin CP: Highly efficient multipotent differentiation of human periodontal ligament fibroblasts induced by combined BMP4 and hTERT gene transfer. Gene Ther 2011, 18:452-461.

doi:10.1186/scrt74

Cite this article as: Maeda $\mathrm{H}$, et al.: Promise of periodontal ligament stem cells in regeneration of periodontium. Stem Cell Research \& Therapy 2011 2:33. 